\title{
Bounds on the electrical resistance between contacting elastic rough bodies
}

\author{
BY J. R. BARBER \\ Department of Mechanical Engineering, University of Michigan, \\ Ann Arbor, MI 48109-2125, USA
}

Received 14 February 2002; revised 14 May 2002; accepted 24 June 2002; published online 1 November 2002

\begin{abstract}
A method is developed for placing bounds on the electrical contact conductance between contacting elastic bodies with rough surfaces. An analogy is first established between contact conductance and the incremental stiffness in the mechanical contact problem. Results from contact mechanics and the reciprocal theorem are then used to bracket the mechanical load-displacement curve between those for two related smooth contact problems. This enable bounds to be placed on the incremental stiffness and hence on the electrical conductance for the rough contact problem. The method is illustrated by two simple examples, but its greatest potential probably lies in establishing the maximum effect of neglected microscales of roughness in a solution of the contact problem for bodies with multiscale or fractal roughness.
\end{abstract}

Keywords: electrical contact resistance; constriction resistance; fractals; surface roughness; contact mechanics

\section{Introduction}

When two elastic bodies are pressed together, the roughness of the surfaces causes the contact to be restricted to a set of microscopic 'actual contact areas'. If we now conduct an electric current between the bodies, the current is constricted to flow through the actual contact areas, causing an additional resistance known as 'constriction resistance' or 'electrical contact resistance' (Holm 1958). Similar considerations apply to the conduction of heat between contacting surfaces (Cooper et al. 1969), though in this case some heat transfer will also occur at other parts of the interface by gas conduction and radiation.

Electrical contact resistance is a problem of considerable technical importance, with applications in resistance welding (Thornton et al. 1997), microelectronic devices, electrical connectors (Bryant 1994) and carbon brushes (Yune \& Bryant 1988). For a broad study of recent developments in the subject, see Slade (1999). The study of electrical contact resistance was pioneered by Holm (1958) and Greenwood (1966), who developed analytical expressions for the resistance due to individual or clustered circular contact areas. The size and spatial distribution of actual contact areas depends on the detailed geometry of the contacting rough surfaces, leading to a complex elastic or elastic/plastic contact problem. There is an extensive body of literature on this subject using, for example, asperity model theories (Greenwood \& Williamson 1966), random process models (Nayak 1971) and fractal 


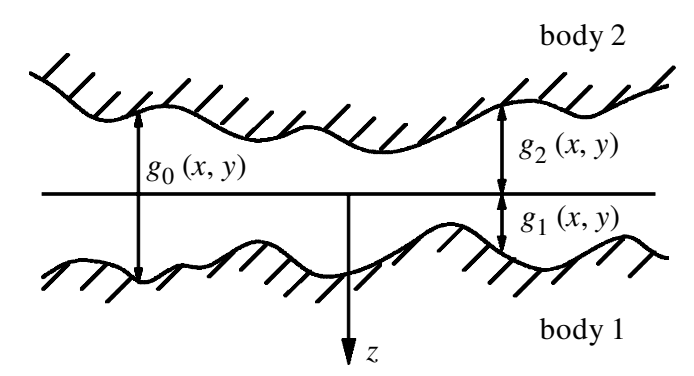

Figure 1. Initial gap between two bodies.

models (Majumdar \& Bhushan 1991; Ciavarella et al. 2000). Earlier models typically assumed that individual actual contact areas were sufficiently widely spaced to be treated as resistances in parallel, or alternatively to be uniformly clustered within a 'nominal contact area' that provided an additional constriction resistance. However, advances in surface measurement show that roughness is a multiscale phenomenon, suggesting that what appears as a single contact on one scale may be resolved as a cluster of smaller contacts on the next smaller scale. This has been graphically demonstrated by Borri-Brunetto et al. (1998), who used a numerical method with progressively refined discretization to solve an elastic contact problem for a surface with fractal characteristics. An analytical investigation of this process for the Weierstrass profile (Ciavarella et al. 2000) suggests that the fractal limit of the actual contact area comprises an infinite set of highly clustered point contacts. It is an open question whether the theoretical contact resistance in this limit would even be bounded.

In the present paper, we shall approach the problem rather differently. Instead of analysing the micromechanics of contact either statistically or deterministically, we seek to impose bounds on the contact resistance in terms of macroscopic properties of the contact geometry and roughness parameters. We first establish an analogy between the electrical conduction problem and the elastic contact problem. We demonstrate that the electrical conductance at any load is proportional to the incremental stiffness in the elastic contact problem. We then use a theorem in contact mechanics to place upper and lower bounds on the contact force required to produce a given level of indentation of a rough surface. These bounds will be shown to be quite close, particularly at high levels of load. It follows that quite good estimates can be determined for the incremental stiffness and hence for the electrical conductance.

\section{The elastic contact problem}

Figure 1 shows two unloaded elastic bodies whose surfaces are defined by the functions $g_{1}(x, y), g_{2}(x, y)$, respectively, relative to a plane datum surface. Although the surfaces are not plane, we assume that surface slopes are sufficiently small for the bodies to be represented by half-spaces in the elastic solution. The functions $g_{1}, g_{2}$ may be used to describe the shape of an indenter, as in the classical Hertzian theory, or for a deterministic description of a rough surface.

The local gap between the bodies in the configuration shown is

$$
g_{0}(x, y)=g_{1}(x, y)+g_{2}(x, y)
$$


Suppose the bodies now experience a relative rigid-body approach $w$ and are simultaneously deformed by surface tractions so as to produce normal surface displacements $u_{z}^{1}(x, y, 0), u_{z}^{2}(x, y, 0)$ in the positive $z$-direction, where superscripts 1 and 2 denote bodies 1 and 2 , respectively. It is clear from figure 1 that a positive displacement $u_{z}^{1}$ will tend to increase the gap, whereas $u_{z}^{2}$ and the rigid-body approach $w$ tend to decrease it. The gap function is therefore modified to

$$
g(x, y)=g_{0}(x, y)-w+u_{z}^{1}(x, y, 0)-u_{z}^{2}(x, y, 0) .
$$

The normal contact pressure $p(x, y)$ is a compressive traction equal on each surface, and hence

$$
p(x, y)=-\sigma_{z z}^{1}(x, y, 0)=-\sigma_{z z}^{2}(x, y, 0),
$$

where we employ the usual sign convention that tensile stresses are positive.

Actual contact can be defined as the condition in which the gap is zero, and hence the contact problem is defined by the unilateral conditions

$$
\begin{array}{ll}
g(x, y)=0 & \text { in } A, \\
p(x, y)=0 & \text { in } \bar{A}, \\
p(x, y)>0 & \text { in } A, \\
g(x, y)>0 & \text { in } \bar{A},
\end{array}
$$

where $A$ denotes the contact area and $\bar{A}$ the remainder of the interfacial plane. Also, since the contact is assumed to be frictionless, we have

$$
\sigma_{z x}^{1}(x, y, 0)=\sigma_{z x}^{2}(x, y, 0)=\sigma_{z y}^{1}(x, y, 0)=\sigma_{z y}^{2}(x, y, 0)=0 \quad \text { in } A \cup \bar{A} .
$$

Boussinesq (1885) showed that the stress and displacement fields in a half-space can be written in terms of harmonic potential functions. In particular, if the halfspace is loaded by purely normal tractions, the solution involves a single harmonic function $\varphi$. This representation is tabulated by Green \& Zerna (1968) and as Solution $\mathrm{F}$ by Barber (1992). At the surface $z=0$, the shear tractions are zero and the normal traction and normal displacement are given by

$$
\begin{aligned}
\sigma_{z z}(x, y, 0) & =-\frac{\partial^{2} \varphi}{\partial z^{2}}(x, y, 0), \\
u_{z}(x, y, 0) & =-\frac{(1-\nu)}{\mu} \frac{\partial \varphi}{\partial z},
\end{aligned}
$$

where $\mu, \nu$ are the modulus of rigidity and Poisson's ratio, respectively.

Using this representation with separate harmonic functions $\varphi_{1}, \varphi_{2}$ for $z>0, z<0$, respectively, we can satisfy the frictionless boundary condition (2.8). The condition of continuity of normal tractions at the interface (2.3) can then be satisfied by imposing the symmetry relation

$$
\varphi_{1}(x, y, z)=\varphi_{2}(x, y,-z) \equiv \varphi(x, y, z), \quad z \geqslant 0 .
$$

Notice that this condition ensures that even $z$-derivatives of the two functions will be equal at $z=0$, but odd $z$-derivatives will be equal and opposite.

Substituting (2.11) into (2.2)-(2.10) reduces the contact problem to a boundaryvalue problem for the function $\varphi(x, y, z)$ in the half-space $z \geqslant 0$, defined by

$$
\nabla^{2} \varphi=0
$$


with

$$
\begin{aligned}
\left(\frac{\left(1-\nu_{1}\right)}{\mu_{1}}+\frac{\left(1-\nu_{2}\right)}{\mu_{2}}\right) \frac{\partial \varphi}{\partial z}(x, y, 0) & =g_{0}(x, y)-w \quad \text { in } A \\
& <g_{0}(x, y)-w \quad \text { in } \bar{A}
\end{aligned}
$$

and

$$
\begin{aligned}
\frac{\partial^{2} \varphi}{\partial z^{2}}(x, y, 0)=0 & \text { in } \bar{A} \\
>0 & \text { in } A .
\end{aligned}
$$

The corresponding total force is then given by

$$
F=\iint_{A} p(x, y) \mathrm{d} A=\iint_{A} \frac{\partial^{2} \varphi}{\partial z^{2}}(x, y, 0) \mathrm{d} A .
$$

This formulation demonstrates the well-known result that the contact problem for two elastic half-spaces is equivalent to that of the indentation of a single half-space of composite modulus,

$$
\frac{1}{M} \equiv \frac{\left(1-\nu_{1}\right)}{\mu_{1}}+\frac{\left(1-\nu_{2}\right)}{\mu_{2}},
$$

by a rigid indenter whose profile is defined by the function $g_{0}(x, y)$ of $(2.1)$. We shall make use of this equivalence to simplify some of the subsequent derivations.

\section{The elastic/electrical analogy}

Suppose that two elastic bodies are pressed together by a force $F$, establishing a contact area $A$. If the contacting bodies are maintained at different electrical potentials $V_{1}, V_{2}$, a current $I$ will flow between them and there will be a contact resistance $R_{\mathrm{e}}$ associated with the constriction of the current through the contact area. If the bodies can be modelled as half-spaces, there is a simple relation between this resistance and the incremental elastic compliance of the contacting bodies. This relation is implicit in the potential function formulation of $\S 2$, but to the best of the author's knowledge, it has never been formally enunciated as a general result.

\section{(a) A boundary-value problem}

We first define a function $\psi(x, y, z)$ in the half-space $z>0$ such that

$$
\nabla^{2} \psi=0
$$

with boundary conditions

$$
\begin{aligned}
\psi=1 \quad & \text { in } A \\
\frac{\partial \psi}{\partial z}=0 & \text { in } \bar{A}
\end{aligned}
$$

with

$$
\psi \rightarrow 0, \quad z \rightarrow \infty
$$


where $A$ is an area (not necessarily connected) on the surface $z=0$ and $\bar{A}$ is the remainder of the surface $z=0$.

This is a well-posed boundary-value problem for any given area $A$. From the solution, we also define the flux density $q(x, y)$ at the surface as

$$
q(x, y)=-\frac{\partial \psi}{\partial z}(x, y, 0)
$$

and the total flux $Q$ as

$$
Q=\iint_{A} q(x, y) \mathrm{d} x \mathrm{~d} y
$$

\section{(b) The electrical problem}

Suppose now that body 1 occupies the region $z>0$ and has resistivity $\rho_{1}$, body 2 occupies $z<0$ and has resistivity $\rho_{2}$ and the two bodies make contact over the area $A$ of the common interface. The electrical potential $V(x, y, z)$ in each body must then satisfy the equation

$$
\nabla^{2} V=0
$$

with boundary conditions

$$
\begin{array}{ll}
V\left(x, y, 0^{+}\right)=V\left(x, y, 0^{-}\right) & \text {in } A, \\
j_{z}\left(x, y, 0^{+}\right)=j_{z}\left(x, y, 0^{-}\right) & \text {in } A, \\
j_{z}\left(x, y, 0^{+}\right)=j_{z}\left(x, y, 0^{-}\right)=0 & \text { in } \bar{A},
\end{array}
$$

where the current density

$$
j_{z}=-\frac{1}{\rho_{i}} \frac{\partial V}{\partial z}, \quad i=1,2,
$$

and is defined to be positive in the direction of the positive $z$-axis.

Also, distant from the contact interface we must have $V(x, y, z) \rightarrow V_{1}$ as $z \rightarrow \infty$ and $V(x, y, z) \rightarrow V_{2}$ as $z \rightarrow-\infty$. Notice that the condition of voltage continuity at the interface (3.8) implies that there is perfect electrical contact between the bodies in the contact area and hence that any contaminant films on the surfaces are sufficiently thin for their electrical resistance to be neglected.

It is easily verified that conditions (3.7)-(3.11) are all satisfied by the potential

$$
\begin{aligned}
V(x, y, z) & =V_{1}+\frac{\rho_{1}\left(V_{2}-V_{1}\right)}{\left(\rho_{1}+\rho_{2}\right)} \psi(x, y, z), \quad z>0, \\
& =V_{2}-\frac{\rho_{2}\left(V_{2}-V_{1}\right)}{\left(\rho_{1}+\rho_{2}\right)} \psi(x, y,-z), \quad z<0,
\end{aligned}
$$

where $\psi$ is the solution of the boundary-value problem of $\S 3 a$. It then follows that the contact area $A$ is an isopotential surface at potential

$$
V(A)=\frac{\rho_{2} V_{1}+\rho_{1} V_{2}}{\left(\rho_{1}+\rho_{2}\right)}
$$

and that the total current transmitted between the two bodies is

$$
I=\iint_{A} j_{z}(x, y, 0) \mathrm{d} A=\frac{\left(V_{2}-V_{1}\right) Q}{\left(\rho_{1}+\rho_{2}\right)},
$$


where $Q$ is defined by (3.6). The electrical contact resistance is therefore

$$
R_{\mathrm{e}}=\frac{\left(V_{2}-V_{1}\right)}{I}=\frac{\left(\rho_{1}+\rho_{2}\right)}{Q} .
$$

\section{(c) The incremental elastic problem}

Consider the case in which the two bodies of $\S 2$ are pressed together by a force $F$, causing a relative approach $w$ and establishing a contact area $A$. Suppose the force is now increased by an infinitesimal increment $\delta F$, producing an infinitesimal increase $\delta w$ (constant) in the relative approach. The incremental problem is therefore defined by the boundary-value problem $\dagger$

$$
\begin{array}{ll}
\frac{\partial \varphi}{\partial z}=-M \delta w & \text { in } A, \\
\frac{\partial^{2} \varphi}{\partial z^{2}}=0 & \text { in } \bar{A},
\end{array}
$$

from $(2.13)-(2.16)$, with

$$
\delta F=\iint_{A} \frac{\partial^{2} \varphi}{\partial z^{2}}(x, y, 0) \mathrm{d} A .
$$

The boundary conditions (3.17), (3.18) can be satisfied by choosing

$$
\frac{\partial \varphi}{\partial z}(x, y, z)=-(M \delta w) \psi(x, y, z)
$$

where $\psi$ is the solution of the boundary-value problem of $\S 3 a$. We then have

$$
\delta F=-M \delta w \int_{A} \frac{\partial \psi}{\partial z}(x, y, 0) \mathrm{d} A=M Q \delta w,
$$

from (3.19) and (3.6). Thus the incremental stiffness is

$$
\frac{\mathrm{d} F}{\mathrm{~d} w}=M Q
$$

(d) Relation between conductance and incremental stiffness

Eliminating $Q$ between (3.22) and (3.16), we obtain

$$
C \equiv \frac{1}{R_{\mathrm{e}}}=\frac{1}{M\left(\rho_{1}+\rho_{2}\right)} \frac{\mathrm{d} F}{\mathrm{~d} w},
$$

showing that contact conductance $C$ is linearly proportional to the incremental stiffness.

\section{Bounds on the elastic contact problem}

Suppose that the nominally plane rough surface of an elastic half-space is described by the function $z=f(x, y)$ and that the half-space occupies the region $z>f(x, y)$,

$\dagger$ There will, in general, also be an increase $\delta A$ in the contact area, but the incremental loading in $\delta A$ will have only second-order effects compared with the rest of the solution as long as $\delta A$ is also infinitesimal. This will always be the case if $A$ is a continuous function of $F$. 


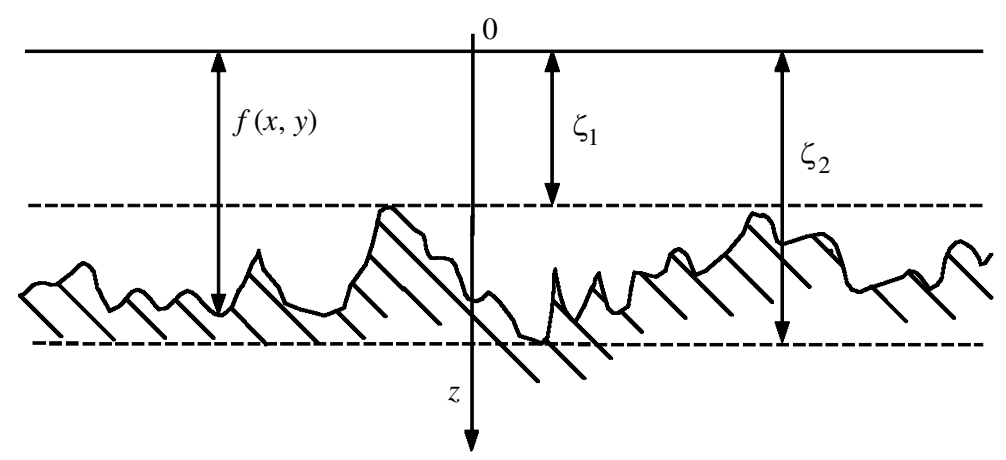

Figure 2. Description of the rough surface.

as shown in figure 2. Since this is a deterministic description, there must be a highest point $\zeta_{1}$ and a lowest point $\zeta_{2}$, defined by

$$
\zeta_{1}=\min (f(x, y)), \quad \zeta_{2}=\max (f(x, y)) .
$$

We next consider two limiting elastic contact problems in which a smooth rigid indenter is pressed into the smooth elastic half-spaces $z \geqslant \zeta_{1}$ and $z \geqslant \zeta_{2}$, respectively. These problems are clearly identical except for a rigid-body displacement. Suppose that the required contact force $F$ can be written

$$
F=F(w)
$$

where $w$ is the rigid-body displacement of the indenter measured from the point of first contact. If the indenter is flat, the contact area will be independent of $w$ and $F(w)$ will be a linear function. For all other cases, the contact area is a non-decreasing function of $w$ (Barber 1974), and consequently the incremental stiffness $\mathrm{d} F / \mathrm{d} w$ is also a non-decreasing function of $w$. The expression (4.2) can be extended to cover the case of non-contact by defining the function $F(w)$ such that $F=0$ for $w<0$.

If the lowest point on the indenter is brought down to the level $\zeta$, the indentation depth for the half-space $z \geqslant \zeta_{1}$ will be $w_{1}=\zeta-\zeta_{1}$ and the corresponding contact force will be

$$
F_{1}(\zeta)=F\left(\zeta-\zeta_{1}\right)
$$

whereas for the half-space $z \geqslant \zeta_{2}$ we have $w_{2}=\zeta-\zeta_{2}$ and

$$
F_{2}(\zeta)=F\left(\zeta-\zeta_{2}\right)
$$

Since $\zeta_{2}>\zeta_{1}$, the monotonicity of $F(w)$ implies that $F_{1}(\zeta)>F_{2}(\zeta)$ for all $\zeta$, as we should expect from simple physical considerations.

In the same way, it is reasonable to expect that the force $F(\zeta)$ required to press the indenter to the same depth $\zeta$ in the rough half-space would be intermediate between these extremes, i.e. $F_{1}(\zeta)>F(\zeta)>F_{2}(\zeta)$. The basis for this expectation is that the force of indentation can only increase if the depth of penetration at any given point either increases or stays the same. However, the result is not self evident, since, for a sufficiently rough surface, there will be regions of separation (non-contact) that are included in the contact area for both of the bounding smooth contact problems. 
To develop a rigorous proof, we first restate the frictionless unilateral contact problem $(2.4)-(2.7)$ as

$$
\begin{array}{ll}
u(x, y)=U(x, y) & \text { in } A, \\
u(x, y)>U(x, y) & \text { in } \bar{A}, \\
p(x, y)=0 & \text { in } \bar{A}, \\
p(x, y)>0 & \text { in } A,
\end{array}
$$

where $u(x, y)=u_{z}(x, y, 0)$ is the inward normal surface displacement of the half-space and $U(x, y)$ is a function describing the shape of the indenter and its indentation. The required theorem can then be stated as follows.

Theorem 4.1. If two distinct frictionless contact problems are defined for the same half-space, in which the respective indentation functions $U_{1}(x, y), U_{2}(x, y)$ satisfy the inequality $U_{1}(x, y) \geqslant U_{2}(x, y)$ for all $x, y$, then the corresponding total forces satisfy the inequality $F_{1} \geqslant F_{2}$.

Proof. Suppose we start with the solution of the contact problem defined by the function $U(x, y)$ and examine the effect of increasing $U$ by a small increment $\delta U(x, y)$ in an infinitesimally small region $\Delta A$ that is part of the original contact area $A$, this function being kept constant in all other regions. We consider this process in two steps. During step $1, u(x, y)$ is increased in $\Delta A$ to restore the contact condition, but the rest of the area $A$ is forced to remain in contact. This may induce tensile tractions in some other region $\Delta A^{\prime}$, which we shall release in step 2. Further applications of step 2 may be required until the inequality constraints are satisfied at all points. We shall show that the total force cannot decrease at either step and hence that the final force exceeds or is equal to the original force. Incremental application of this procedure to all regions $\Delta A$ establishes the theorem.

Step 1. During step 1, the incremental process involves the application of a contact pressure $p_{1}(x, y)$ in $\Delta A$, while the remainder of the area $A-\Delta A$ experiences zero normal displacement. This problem is defined by the boundary conditions

$$
\begin{array}{rlrl}
u_{1}(x, y) & =\delta U(x, y) & & \text { in } \Delta A, \\
& =0 & & \text { in } A-\Delta A, \\
p_{1}(x, y)=0 & & \text { in } \bar{A} .
\end{array}
$$

We also define an auxiliary problem through the boundary conditions

$$
\begin{array}{ll}
u_{2}(x, y)=1 & \text { in } A, \\
p_{2}(x, y)=0 & \text { in } \bar{A} .
\end{array}
$$

Applying Betti's reciprocal theorem to these two problems, we obtain the relation

$$
\iint_{A+\bar{A}} p_{1}(x, y) u_{2}(x, y) \mathrm{d} x \mathrm{~d} y=\iint_{A+\bar{A}} p_{2}(x, y) u_{1}(x, y) \mathrm{d} x \mathrm{~d} y .
$$

Substituting for $p_{1}, u_{1}, p_{2}, u_{2}$ from (4.9)-(4.13), we obtain

$$
\delta F \equiv \iint_{A} p_{1}(x, y) \mathrm{d} x \mathrm{~d} y=\iint_{\Delta A} p_{2}(x, y) \delta U(x, y) \mathrm{d} x \mathrm{~d} y,
$$

where $\delta F$ is the total indentation force in the incremental problem. 


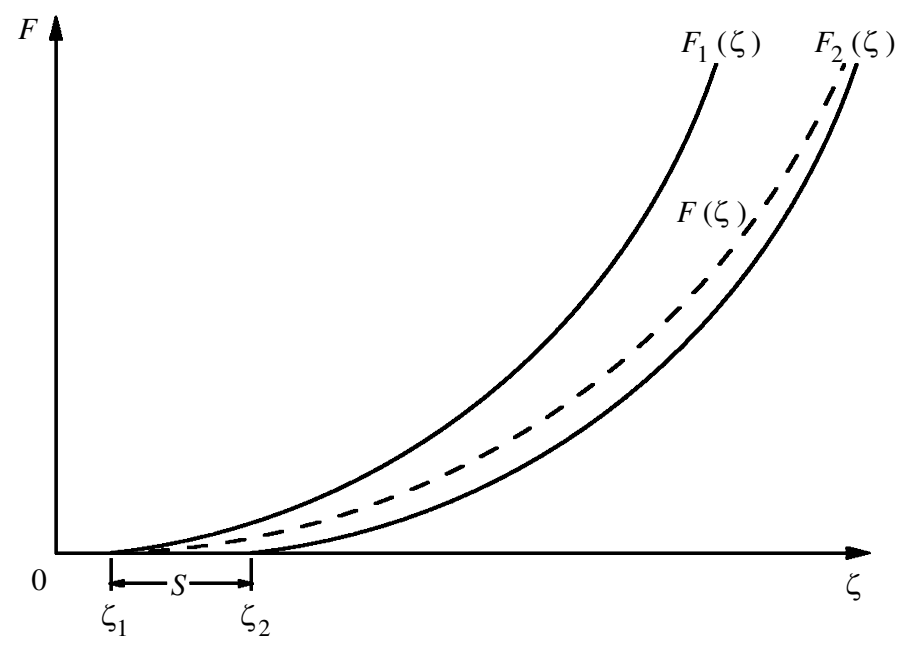

Figure 3. Typical functions $F_{1}(\zeta), F_{2}(\zeta)$. The load-displacement relation $F(\zeta)$ for the rough surface must lie between these two curves.

Now, in a frictionless indentation problem for the half-space, the maximum normal surface displacement must occur in an area of positive contact pressure (Barber 1974), and in the auxiliary problem the only loaded area is $A$, throughout which $u_{2}=1$. It follows that all points in $A$ are points of maximum $u_{2}$ and hence that $p_{2}(x, y)>0$ for all points in $A$. We also have $\delta U(x, y)>0$ ex hypothesi, and hence the integrand on the right-hand side of (4.15) is positive for all $x, y$, from which

$$
\delta F>0 .
$$

In other words, the indentation force $F$ must increase during step 1.

Step 2. During step 2, we release the tensile traction in some small area $\Delta A^{\prime}$, keeping the rest of the contact area $A$ unchanged. The superposed traction in $\Delta A^{\prime}$ during this step is therefore compressive, and the incremental problem is identical with step 1 , except that $\Delta A^{\prime}$ replaces $\Delta A$ and the incremental force is prescribed in $\Delta A^{\prime}$ instead of the incremental displacement. The above argument therefore carries over to this step, showing that the indentation force cannot decrease during step 2 and hence establishing the theorem.

\section{Bounds on the contact conductance}

Figure 3 shows a plot of typical functions $F_{1}(\zeta), F_{2}(\zeta)$ from (4.3) and (4.4). Both these expressions are derived from the same function $F(w)$, and hence the two curves must have the same shape. They are merely separated by a constant distance,

$$
s=\zeta_{1}-\zeta_{2},
$$

along the $\zeta$-axis. Notice also that the derivative $\mathrm{d} F / \mathrm{d} \zeta$ must be a non-decreasing function of $\zeta$, so the curves will be concave upwards except in the limiting case of a flat indenter, for which they will be parallel straight lines.

The theorem of $\S 4$ shows that the function $F(\zeta)$ defining the load-displacement relation for the indentation of the rough elastic half-space must lie between the two 


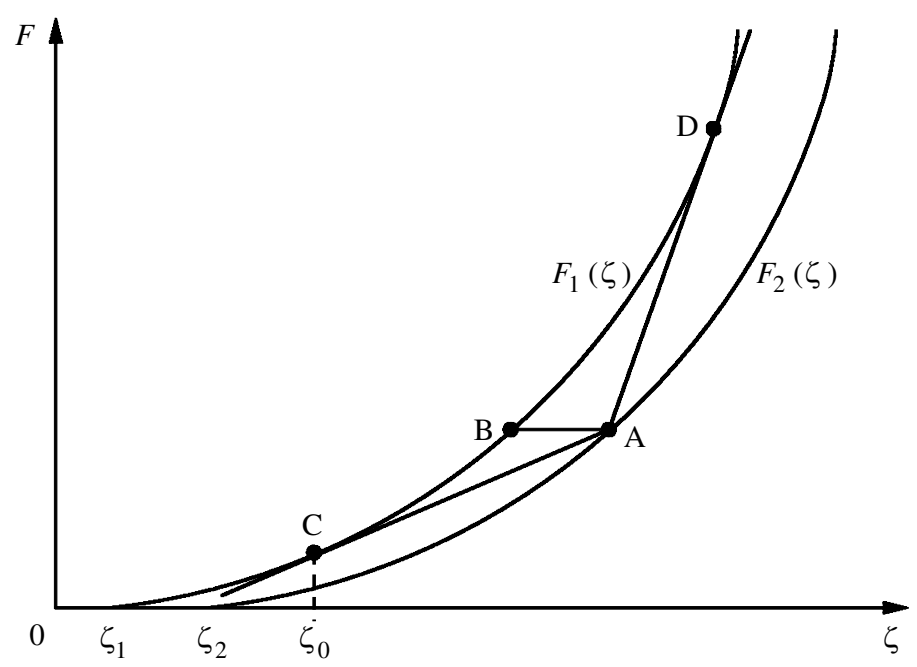

Figure 4. Construction for determining bounds on the incremental stiffness.

curves in figure 3. It must also be concave upwards. Contact will start as soon as $\zeta>\zeta_{1}$, and the proportion of the nominal contact area in actual contact will increase with $F$, so we anticipate a curve that starts from the beginning of the $F_{1}$ curve at $\left(\zeta_{1}, 0\right)$ and moves closer to the $F_{2}$ curve with increasing $F$. A plausible curve is shown dashed in figure 3 .

A lower bound on the slope of the $F(\zeta)$ curve at a given value of $F$ can be determined by the construction of figure 4 . For a given force $F$, we start at the point $\mathrm{A}$ on the $F_{2}$ curve and draw the tangent $A C$ to the $F_{1}$ curve. The slope $\mathrm{d} F / \mathrm{d} \zeta$ at $F$ cannot be less than the slope of this line, since if we start anywhere between the points $\mathrm{A}$ and $\mathrm{B}$ with a line of lesser slope and extend it backwards towards $F=0$, we could only stay between the lines $F_{1}(\zeta)$ and $F_{2}(\zeta)$ by making the line convex upwards, which violates the condition that the incremental stiffness $\mathrm{d} F / \mathrm{d} \zeta$ be a non-decreasing function of $\zeta$.

By a similar argument, it follows that the slope $\mathrm{d} F / \mathrm{d} \zeta$ at a given value of $F$ must be lower than that of the tangent line $\mathrm{AD}$ in figure 4 , since a steeper line than this could only stay within the bounding curves if it were convex upwards.

Once these limiting slopes have been determined, the corresponding upper and lower bounds on conductance follow immediately from (3.23).

\section{(a) Contact of two rough bodies}

These arguments are readily extended to the contact of two rough elastic bodies. As shown in $\S 2$, the contact problem depends only on the sum $g_{0}$ of the two profile functions $g_{1}, g_{2}$. Thus, we can 'transfer' the roughness of one body to the other, obtaining a composite roughness description

$$
f(x, y)=f_{1}(x, y)+f_{2}(x, y) .
$$

The required roughness height parameter $s$ is then defined as before as

$$
s=\max (f(x, y))-\min (f(x, y)) .
$$




\section{Examples}

\section{(a) Spherical indenter}

Suppose that the indenter is a sphere of radius $R$. The results for the smooth contact problem then follow from the classical Hertzian theory. The load-displacement relation is

$$
F=C_{1} w^{3 / 2},
$$

where

$$
C_{1}=\frac{8}{3} M R^{1 / 2},
$$

(Johnson 1985) and the corresponding incremental stiffness is

$$
\frac{\mathrm{d} F}{\mathrm{~d} w}=\frac{3}{2} C_{1} w^{1 / 2} .
$$

If we choose a coordinate system such that $\zeta_{1}=0$ and denote the point $\mathrm{C}$ in figure 4 by $\left(\zeta_{0}, C_{1} \zeta_{0}^{3 / 2}\right)$, the equation of the line CA must then be

$$
F=C_{1} \zeta_{0}^{3 / 2}+\frac{3}{2} C_{1} \zeta_{0}^{1 / 2}\left(\zeta-\zeta_{0}\right),
$$

and its intersection with the line

$$
F_{2}(\zeta)=C_{1}(\zeta-s)^{3 / 2}
$$

occurs when

$$
(\zeta-s)^{3 / 2}=\frac{3}{2} \zeta_{0}^{1 / 2} \zeta-\frac{1}{2} \zeta_{0}^{3 / 2}
$$

Introducing the dimensionless variables

$$
x=\sqrt{\frac{\zeta-s}{\zeta_{0}}}, \quad S=\frac{s}{\zeta_{0}},
$$

this equation can be written in the form

$$
S=\frac{2}{3} x^{3}-x^{2}+\frac{1}{3}=\frac{2}{3}\left(x+\frac{1}{2}\right)(x-1)^{2} .
$$

We can also use this notation to define the dimensionless force and incremental stiffness as

$$
F^{*} \equiv \frac{F}{M \sqrt{R s^{3}}}=\frac{8 x^{3}}{3 S^{3 / 2}}, \quad K^{*} \equiv \frac{1}{M \sqrt{R s}} \frac{\mathrm{d} F}{\mathrm{~d} \zeta}=\frac{4}{\sqrt{S}} .
$$

We then use (3.23) to obtain the dimensionless electrical contact conductance $C^{*}$, defined as

$$
C^{*} \equiv \frac{\left(\rho_{1}+\rho_{2}\right) C}{\sqrt{R s}}=\frac{4}{\sqrt{S}} .
$$

Equation (6.8) determines the value of $\zeta$ at the intersection point $\mathrm{A}$ in figure 4 corresponding to the tangent point defined by the value $\zeta_{0}$. The solution defines a lower bound (minimum slope) if $\mathrm{C}$ is to the left of $\mathrm{B}$, and hence $(\zeta-s)>\zeta_{0}$ and $x>1$. Conversely, upper-bound intersections correspond to $0<(\zeta-s)<\zeta_{0}$ and $0<x<1$. 


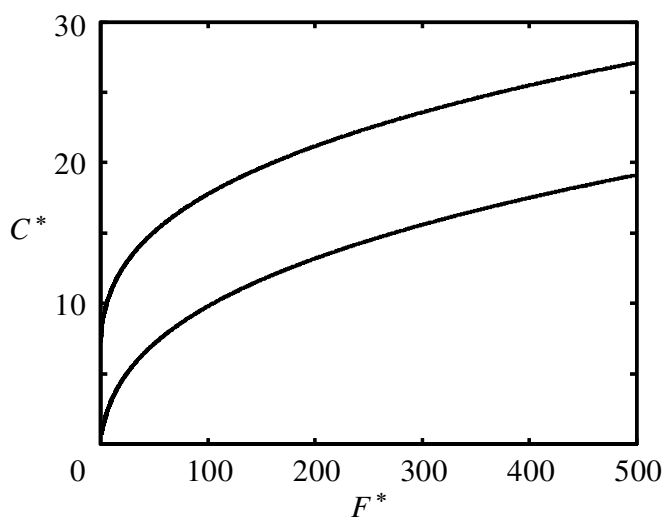

Figure 5. Upper and lower bounds of dimensionless conductance $C^{*}$ for the rough spherical indenter.

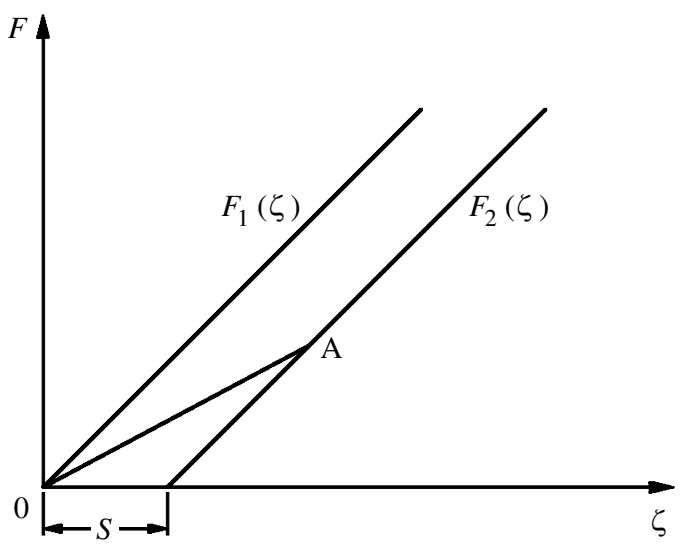

Figure 6. Construction for determining the lower bound for the flat indenter problem.

Equation (6.8) is cubic in $x$ and can therefore be solved in closed form, but it is more convenient to construct the bounds parametrically. Thus, for a given value of $x$ in $x>0$, we calculate $S$ from (6.8) and then obtain $F^{*}$ and $C^{*}$ from (6.9) and (6.10). By following this procedure for all values in the range $0<x<\infty$, the entire upper and lower bounding curves can be constructed. Figure 5 shows the upper and lower bounds for $C^{*}$ as a function of $F^{*}$.

\section{(b) Flat cylindrical indenter}

If the indenter is a rigid perfectly conducting flat-ended cylinder of radius $a$, the load-displacement relation is

$$
F=C_{2} w
$$

where

$$
C_{2}=\frac{4 \mu a}{(1-\nu)}
$$

and the incremental stiffness is

$$
\frac{\mathrm{d} F}{\mathrm{~d} w}=C_{2},
$$


where $\mu, \nu$ are the elastic properties of the half-space material (Barber 1992). The functions $F_{1}(\zeta), F_{2}(\zeta)$ now define parallel straight lines, as shown in figure 6 , and the upper bound in this case must correspond to a line of slope $C_{2}$. The lower bound is defined by the line OA, which has slope

$$
K=\frac{F}{\zeta_{A}}=\frac{F}{F / C_{2}+s},
$$

and the corresponding bounds on the conductance $C$ are easily shown to be

$$
\frac{4 a}{\rho}>C>\frac{4 a}{\rho} /\left(1+\frac{4 \mu a s}{F(1-\nu)}\right) .
$$

In this simple case, the upper bound on $C$ is identical with Holm's expression for the conductance through a circular contact of radius a (Holm 1958) and corresponds to complete electrical contact inside the plan form of the indenter. More generally, for a rigid flat-ended indenter of any shape, the bounding curves $F_{1}(\zeta)$ and $F_{2}(\zeta)$ will be parallel straight lines, and hence the upper bound for any such indenter will correspond to complete electrical contact inside the plan form.

\section{Consequences for fractal rough surfaces}

The bounds established in $\S 5$ bracket the electrical contact conductance between two finite and non-zero values as long as the surface description has a finite maximum peak-to-valley height $s$. This condition must be satisfied by any deterministically defined fractal-surface description, such as the Weierstrass profile investigated by Ciavarella et al. (2000) and others. We conclude that the electrical conductance between contacting perfectly elastic fractal surfaces must be finite and non-zero, despite the indication that the theoretical contact area in this case comprises an infinite set of point contacts.

As the elastic contact process is extended to smaller and smaller scales, the total contact area decreases and the mean contact pressure increases (Borri-Brunetto et al. 1998). We must therefore anticipate that plastic deformation or some other failure mechanism will dominate the contact process at sufficiently small scales, probably giving contact areas of finite number and size. However, for materials of high yield strength, it is possible that the contact conductance will have converged to its limit before the 'plastic scale' is reached and hence that a simpler elastic prediction may be sufficiently accurate.

The function $g_{0}(x, y)$ of $(2.1)$ defines both the macroscopic shape of the contacting bodies and the superposed surface roughness, but the distinction between these quantities is essentially arbitrary. Thus, for a multiscale surface, we could envisage solving for the relation $F(w)$ for an indenter whose shape explicitly includes the coarser scales of the surface roughness (e.g. by an asperity model theory), leaving only the finer scales to be described by the function $f(x, y)$ of $(4.1)$. This would reduce the value of $s$ and tighten the resulting bounds. In other words, the methodology of $\S 5$ can be seen as placing bounds on the effect of those microscales of roughness not accounted for in a classical solution of the contact problem with roughness. 


\section{Conclusions}

We have used an analogy between electrical conductance and elastic contact to develop a methodology for placing bounds on the electrical conductance between two contacting elastic bodies with rough surfaces. The bounds depend only on properties of the corresponding smooth contact problem and the maximum peak-to-valley height of the combined roughness.

The method is illustrated by two simple examples, but its greatest potential probably lies in establishing the maximum effect of neglected microscales of roughness in a solution of the contact problem for bodies with multiscale or fractal roughness.

\section{References}

Barber, J. R. 1974 Determining the contact area in elastic indentation problems J. Strain Analysis 9, 230-232.

Barber, J. R. 1992 Elasticity. Dordrecht: Kluwer.

Borri-Brunetto, M., Carpinteri, A. \& Chiaia, B. 1998 Lacunarity of the contact domain between elastic bodies with rough boundaries. In Probamat-21st century: probabilities and materials (ed. G. Frantziskonis), pp. 45-64. Dordrecht: Kluwer.

Boussinesq, J. 1885 Application des potentiels á l'étude de l'équilibre et du mouvement des solides élastiques. Paris: Gauthier-Villars.

Bryant, M. D. 1994 Resistance buildup in electrical connectors due to fretting corrosion of rough surfaces. IEEE Trans. Comp. Packaging Manufact. Technol. A 17, 86-95.

Ciavarella, M., Demelio, G., Barber, J. R. \& Jang, Y. H. 2000 Linear elastic contact of the Weierstrass profile. Proc. R. Soc. Lond. A 456, 387-405.

Cooper, M. G., Mikic, B. B. \& Yovanovich, M. M. 1969 Thermal contact conductance. Int. J. Heat Mass Transfer 12, 279-300.

Green, A. E. \& Zerna, W. 1968 Theoretical elasticity, 2nd edn, § 5.7. Oxford: Clarendon.

Greenwood, J. A. 1966 Constriction resistance and the area of real contact. Br. J. Appl. Phys. 17, 1621-1632.

Greenwood, J. A. \& Williamson, J. B. P. 1966 The contact of nominally flat surfaces. Proc. $R$. Soc. Lond. A 295, 300-319.

Holm, R. 1958 Electrical contacts handbook. Springer.

Johnson, K. L. 1985 Contact mechanics. Cambridge University Press.

Majumdar, A. \& Bhushan, B. 1991 Fractal model of elastic-plastic contact between rough surfaces. ASME J. Tribol. 113, 1-11.

Nayak, P. R. 1971 Random process model of rough surfaces. ASME J. Lubrication Technol. 93, 398-407.

Slade, P. G. 1999 Electrical contacts-principles and applications. New York: Dekker.

Thornton, P. H., Krause, A. R. \& Davis, R. G. 1997 Contact resistances in spot welding. Weld. J. 75, 401s-412s.

Yune, Y. G. \& Bryant, M. D. 1988 Transient nonlinear thermal runaway effects in carbon graphite electrical brushes. IEEE Trans. Comp. Hybrids Manufact. Technol. 11, 91-100. 